\title{
The predictive value of tissue Doppler for left ventricular recovery and remodeling after primary percutaneous coronary intervention
}

\begin{abstract}
Background: The main goal in the management of ST segment elevation myocardial infarction (STEMI) is an early restoration of coronary artery flow in order to preserve viable myocardium. Doppler tissue imaging (DTI) is a simple modality that measures tissue velocity during cardiac cycle.

Objective: To assess the role of DTline valuation of LV recovery after PCI.

Methods: Fifty patients were included in this study with STEMI, and were candidate for primary PCI. Cardiac enzymes and ECG were done during admission and 12 hours after PCI for assessment of noninvasive criteria for reperfusion. Echocardiography with DTI was performed for all patients to assess $\mathrm{LV}$ recovery and remodeling at baseline and one month after PCI. Primary PCI was performed and patients were divided into two groups: Group A with good reperfusion, and group B with poor reperfusion.

Results: In patients of group A (78\%), there was a statistically significant improvement of the LV EF more than $5 \%$ one month after primary PCI (LV recovery) $(\mathrm{p}<0.001)$, while in group B, there was a statistically significant increase in LVED volume more than $20 \%$ (LV remodeling) $(\mathrm{P}<0.01)$. The mean systolic $(\mathrm{S})$ velocity showed a cutoff value of $4.83 \mathrm{~cm} / \mathrm{sec}$ or more for prediction of recovery of global systolic function with a sensitivity and specificity of $100 \%$ and $90 \%$ respectively. Out of 11 patients in group B, 3 patients (27.27\%) developed MACE and admitted to CCU, 2 of them with heart failure, while other one needed revascularization.
\end{abstract}

Conclusion: LV recovery occurs in STEMI patients treated with primary PCI and it is an important for favorable outcome.

Keywords: LV remodeling after primary PCI, tissue doppler imaging, myocardial infarction, preservation of left ventricular
Volume I Issue 6 - 2014

\author{
Hesham Rashid,' Hesham Abu El-Enien,' \\ Maher Ibraheem ${ }^{2}$ \\ 'Cardiology department, Benha University, Egypt \\ ${ }^{2}$ National Heart Institute, Cairo, Egypt
}

Correspondence: Hesham Khalid Rashid Mousa, Cardiology Department, Benha University, Egypt, Tel 201014977666, Email hesham_mosa200I@yahoo.com

Received: November 13, 2014 | Published: November 28, 2014
Abbreviations: Áa, late diastolic velocity; Éa, early diastolic

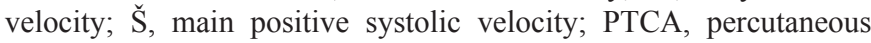
transluminal coronary angiography; PW-DTI, pulsed wave doppler tissue imaging; MBG, myocardial brush grade; MACE, major advanced cardiac events

\section{Introduction}

The main goal in the management of acute myocardial infarction (AMI) is an early restoration of coronary artery flow in order to preserve viable myocardium. Primary percutaneous coronary intervention (PCI) has proven to be superior to other reperfusion strategies in terms of mortality reduction and preservation of left ventricular (LV) function. ${ }^{1}$ Despite improvements in the treatment of MI, $30 \%$ of patients show LV remodeling post-MI, moreover, it is expected that almost $30 \%$ will have an LV ejection fraction (LVEF) below $40 \%$ at 6 months postMI. ${ }^{2}$ Doppler tissue imaging is a modality developed in last years that measures tissue velocity during cardiac cycle. It has been previously demonstrated that there is a significant relationship between successful reperfusion after thrombolytic therapy in acute myocardial infarction as indicated by the conventional noninvasive reperfusion criteria and pulsed wave tissue Doppler (PTD) parameters. ${ }^{3}$ The study aimed to assess the role of pulsed wave tissue Doppler in evaluation of left ventricular recovery and remodeling after primary PCI in relation to other invasive and non invasive reperfusion criteria.

\section{Material}

The study included 50 consecutive patients selected randomly and admitted at CCU of Benha University Hospital and National Heart Institute by acute ST-elevated myocardial infarction and were candidate for PCI. Patients with past history of IHD, patients received thrombolytic therapy, patients with valvular disease, cardiomyopathy, significant left ventricular hypertrophy, pericardial disease, or patients with abnormal ECG as, BBB, pre-excitation, AF, atrial flutter, or complete AV block, or patients underwent coronary artery bypass surgery were excluded from the study.

Study design: All patients were subjected to the following:

Informed consent from all participants was obtained. Full history taking with especial attention on the risk factors e.g., age, gender, smoking, presence of Diabetes Mellitus, dyslipidaemia, hypertension, family history of IHD, and previous myocardial infarctions.

\section{Full clinical examination}

Electrocardiographic analysis: serial S-T segment analysis before and at the end of the coronary intervention was done by one observer blinded to clinical data. The sum of S-T segment elevations was measured manually 20 milliseconds after the end of the QRS complex. Adequate resolution of S-T segment elevation after successful re- 
canalization was expressed as a percentage of the initial S-T segment elevation. S-T segment resolution $<50 \%$ was defined as a marker of impaired microvascular reperfusion. On the other hand, S-T segment resolution greater than $50 \%$ indicated good myocardial reperfusion. ${ }^{4}$

Biochemical markers: creatine phosphokinase (CK)-MB and total $\mathrm{CK}$ were done as biochemical markers of myocardial reperfusion every 6 hours for 24 hours, then daily after PCI. Serum CK peaking within the first 12 hours of PCI was considered a noninvasive marker of reperfusion. ${ }^{5}$

Routine laboratory investigations: Including $\mathrm{CBC}$, liver functions, kidney functions and coagulation profile were done for each patient during hospitalization.

\section{Coronary intervention}

a) All patients received $600 \mathrm{mg}$ loading dose of clopidogrel before PCI.

b) After informed consent, Coronary angiography (transfemoral approach) was performed as soon as possible starting by catheterization of the artery of the non-infarct region, followed by the culprit one. PTCA \pm stenting of the culprit lesion(s) was done with 10,000 units of heparin (UFH) given intravenously just prior to the intervention.

c) The decision to use glycoprotein IIb/IIIa receptor inhibitors was taken when indicated.

Invasive reperfusion criteria: Gibson et al. ${ }^{6}$

TIMI flow grades (TFGs): It is an angiographic marker for successful reperfusion. Myocardial perfusion was described using myocardial blush grades (MBG). Patients with MBG 0 or 1 indicate poor reperfusion while patients with MBG 2 or 3 , were considered to be angiography successfully re-perfused. ${ }^{7}$ TIMI flow and MBG were visually assessed on the angiogram and described immediately after the primary PCI by the performing cardiologist. According to the noninvasive reperfusion criteria (ECG analysis and cardiac markers) and the invasive reperfusion criteria (TIMI flow grades and MBG), all patients were divided into two groups:

I. Group (A): included 39 patients with good reperfusion criteria.

II. Group (B): included 11 patients with poor reperfusion criteria.

Echocardiography and Pulsed tissue Doppler examination

All patients were evaluated using Philips echocardiography machines within 2 days of AMI, and one month later using the same protocol and all parameters were blinded to the results of the primary PCI.

I. Standard echocardiography examination: Edvardsen et al. ${ }^{8}$

i. Assessment of LV size and function:

a. LV volumes were obtained from the apical window to get LVEDV, LV end-systolic volume (LVESV) and measuring EF.

b. LV functional recovery was defined as an increase of $5 \%$ or more of the calculated LV EF between the first and the second echocardiographic evaluation.

c. LV remodeling was defined as increase in LVEDV $>20 \%$ or LVESV $>15 \%$.

ii. Assessment of regional LV function through wall motion score index. It was measured as the sum of segmental scores $(1=$ normal, 2=hypokinesia, $3=$ akinesia, 4=dyskinesia) divided by the number of segments visualized.

II. Pulsed tissue Doppler examination (PTD): Three major velocities were recorded for assessing the PW-TVI spectral display: velocity S wave $(\mathrm{cm} / \mathrm{sec})$, Ea wave $(\mathrm{cm} / \mathrm{sec})$, and velocity Aa wave $(\mathrm{cm} / \mathrm{sec})$.

\section{Follow up the patients}

All patients discharged after PCI with the same anti-ischemic drugs, ACEI, beta blocker and regularly observed at outpatient clinic and contacted with his family by telephone for development of any major adverse cardiac events (MACE) as recurrent chest pain, needs for hospitalization, or reperfusion and lastly for death.

\section{Statistics}

All data analyses were performed with software (Statistical Package for Social Sciences SPSS for Windows 12.0, SPSS Inc, and Chicago IL). Continuous variables between groups were compared by the Student test. The correlation between changes in systolic PTD parameters and global EF were calculated using Pearson's correlation coefficient. Receiver operating characteristic curves were constructed to detect the best cut-off value for a change in mean S-wave velocity to detect LV functional recovery. The sensitivities, specificities, and positive and negative predictive values for the various markers of reperfusion in detecting LV functional recovery were calculated. A probability value $(\mathrm{P}$-value $<0.05)$, (2-tailed) was considered significant results are expressed as mean $\pm \mathrm{SD}$, unless otherwise specified. ${ }^{9}$

\section{Results}

Patients enrolled in this study were divided into two groups:

I. Group A: Included 39 patients (78\%) with good reperfusion criteria \{invasive (TIMI 3 Flow and MBG 2or 3) and noninvasive (ST-segment resolution $\geq 50 \%$ and early peaking of cardiac biomarkers)\}.

II. Group B: Included 11 patients $(22 \%)$ with poor reperfusion criteria \{invasive (TIMI 0,1 or 2 Flow and MBG 0 or 1 ) and non-invasive (ST-segment resolution $<50 \%$ and absence of early peaking of cardiac biomarkers)\}.

Demographic data of the studied groups (Table 1) There was no significant difference between the two groups as regard age distribution. The Mean age \pm SD was $50.4 \pm 9$.8years in group A and $51.7 \pm 14.2$ years in group $\mathrm{B}(\mathrm{P}>0.05)$, out of thirty nine patients within group A, twenty five patients $(64.1 \%)$ were males and fourteen patients $(35.9 \%)$ were females versus three males $(27.2 \%)$ and eight females $(72.7 \%)$ in group B with a significant difference between the studied groups. Clinical examination, history taking and laboratory investigations revealed that presence of risk factors of CAD has been almost a constant finding in all patients. There is significant higher incidence of DM (100\%), HTN (72.7\%), and positive family history of CAD $(72.7 \%)$ in group B compared to group A ( $p$ value $<0.05$ ). Coronary Angiography and Primary PCI Data (Table 2) Fifty percent of patients were presented with anterior MI, while the other fifty were diagnosed as inferior MI. Fifty percent of patients had LAD lesion, while the RCA was occluded in $38 \%$ of patients and the LCX was the culprit in $12 \%$ of patients with no significant difference between both groups as regard distribution of the culprit vessel. Fourteen patients $(35.9 \%)$ in group A had single vessel disease versus 5 patients $(45.46 \%)$ in group B, also 14 patients (35.9\%) in group A had 2-vessel 
disease versus 4 patients $(36.36 \%)$ in group B, and only 11 patients $(28.28 \%)$ in group A had 3-vessel disease versus two patients in group B.

PCI with stent of culprit vessel was achieved in all patients. Bare metal stents were more commonly $(76 \%)$ deployed at the culprit lesion than drug-eluting stents $(24 \%)$, and more commonly used in group B $(90.9 \%)$ than in group A $(71.7 \%)$. All patients in group A were successfully re-perfused at the tissue level achieving MBG 2 (48.72\%) and MBG 3(51.28\%), while no patients in group $\mathrm{B}$ achieved successful reperfusion (MBG 0 in $45.45 \%$ of group B patients and MBG 1 in $54.55 \%$ of patients). All patients in group A were successfully re-canalized achieving TIMI 3 flow grade(about $78 \%$ of entire study group of patients) while no patients in group B achieved it. The predictors of failure re-canalization were, DM, HTN, and patients had positive family history of CAD.

Table I Demographic data among the studied groups

\begin{tabular}{llll}
\hline & $\begin{array}{l}\text { Group A } \\
\text { N=39 }\end{array}$ & Group B & P value \\
& N=I I & \\
\hline Mean Age \pm SD & $50.4 \pm 9.8$ & $51.7 \pm 14.2$ & $>0.05$ \\
Female & $14(35.9 \%)$ & $8(72.7 \%)$ & $<0.05^{*}$ \\
Male & $25(64.1 \%)$ & $3(27.3 \%)$ & \\
DM & $19(48.72 \%)$ & 1 I $(100 \%)$ & $<0.00 I^{*}$ \\
HTN & $19(48.72 \%)$ & $8(72.73 \%)$ & $<0.05^{*}$ \\
dyslipidemia & $23(58.97 \%)$ & $6(54.55 \%)$ & $>0.05$ \\
Smoking & $14(35.90 \%)$ & $2(18.18 \%)$ & $>0.05$ \\
Family history & $15(38.46 \%)$ & $8(72.73 \%)$ & $<0.05^{*}$
\end{tabular}

Table 2 Coronary angiography and $\mathrm{PCl}$ data of both groups

\begin{tabular}{|c|c|c|c|c|c|}
\hline & \multirow{2}{*}{$\begin{array}{l}\text { Group A } \\
\begin{array}{l}\text { N=39 } \\
(\mathbf{7 8} \%)\end{array}\end{array}$} & \multirow{2}{*}{$\begin{array}{l}\text { Group } \\
B \\
N=1 \mid \\
(22 \%)\end{array}$} & \multicolumn{2}{|c|}{ Chi-Square } \\
\hline & & & & $\mathbf{X} 2$ & P-value \\
\hline \multirow[t]{3}{*}{ Culprit vessel } & LAD & $20(51.28)$ & $5(45.45)$ & 3.972 & 0.137 \\
\hline & LCX & $6(15.38)$ & $0(00)$ & & \\
\hline & $\mathrm{RCA}$ & $13(33.33)$ & $6(54.55)$ & & \\
\hline \multirow{3}{*}{$\begin{array}{l}\text { Number } \\
\text { of diseased } \\
\text { vessels }\end{array}$} & I-vesseles & $14(35.90)$ & $5(45.46)$ & 0.558 & 0.756 \\
\hline & 2 -vesseles & $14(35.90)$ & $4(36.36)$ & & \\
\hline & 3 -vesseles & II (28.20) & $2(18.18)$ & & \\
\hline \multirow[t]{2}{*}{ Type of stent } & $\begin{array}{l}\text { Bare } \\
\text { metal }\end{array}$ & 28 (7I.79) & $10(90.9)$ & 2.005 & 0.157 \\
\hline & $\begin{array}{l}\text { Drug- } \\
\text { eluting }\end{array}$ & II (28.2I) & I (9.I) & & \\
\hline \multirow{4}{*}{$\begin{array}{l}\text { TIMI flow } \\
\text { grade }\end{array}$} & Grade 0 & $0(00)$ & $3(27.27)$ & 52.69 & $<0.001 *$ \\
\hline & Grade I & $0(00)$ & $4(36.36)$ & & \\
\hline & Grade 2 & $0(00)$ & $4(36.36)$ & & \\
\hline & Grade 3 & $39(100)$ & $0(00)$ & & \\
\hline \multirow{2}{*}{\multicolumn{2}{|c|}{ Myocardial blush grade }} & $\begin{array}{l}\text { Grade } \\
2=19(48.7)\end{array}$ & \multicolumn{3}{|c|}{ Grade $0=5$ (45.5) } \\
\hline & & $\begin{array}{l}\text { Grade 3= } \\
20(51.3)\end{array}$ & \multicolumn{3}{|c|}{ Grade I =6 (54.5) } \\
\hline
\end{tabular}

\section{Echocardiography}

Standard echocardiography: i. In patients with group A (78\%), there was a statistically significant improvement of LV EF more than $5 \%$ (recovery), it was $(46.8 \pm 6.3)$ at baseline and became $(59.3 \pm 6.2)$ one month after PCI $(\mathrm{p}<0.001)$, and this is reflected on significant improvement of resting wall motion score index after one month follow up $(\mathrm{p}<0.05)$.

ii. In patients with group B $(22 \%)$, there was a statistically significant increase $>20 \%$ in the mean of LVED volume (LV remodeling) from $(165.4 \pm 15.6)$ at baseline to $(195.4 \pm 16.3)$ one month after PCI $(\mathrm{P}<0.01)$.

Tissue Doppler data: Both systolic and diastolic PTD parameters were recorded from 6 sectors (anterior, inferior, anteroseptum, inferoseptum, posterior and lateral walls).

i. A-Systolic function (S wave)

a. When the mean value of the (S) wave in group A at baseline (5.5 \pm 0.28$)$ was compared to the same group one month after PCI (9.9 \pm 0.72$)$; there was significant improvement in the mean $(\mathrm{S})$ wave value $(\mathrm{P}<0.01)$, while it was reduced in group B $(\mathrm{p}>0.05)$.

ii. B-Diastolic function parameters

a. Ea wave: there was significant improvement in the mean Ea wave value of group A after PCI $(10.7 \pm 0.83)$ compared to $(6.3 \pm 0.64)$ at baseline $(\mathrm{P}<0.01)$, while there was significant reduction in the mean Ea wave velocity of group $\mathrm{B}$, it was $(5.5 \pm 0.67)$ at baseline and became $(3.6 \pm 0.69)$ after PCI $(\mathrm{p}<0.05)$.

b. *Aa wave: there was insignificant correlation between the mean values of a wave velocity of both groups at baseline and after PCI ( $p>0.05)$. *E / A ratio: it was significantly improved after PCI in group A $(p<0.01)$, while it was reduced after PCI on group $B(p>0.05)$.

\section{Detection of LV functional recovery}

In comparison between group A and group B using the mean systolic (S) velocity showed a cutoff value of $4.83 \mathrm{~cm} / \mathrm{sec}$ or more for prediction of recovery of global systolic function with a sensitivity and specificity of $100 \%$ and $90 \%$ respectively while the positive predictive value, negative predictive value and accuracy were $97.5 \%$, $100 \%$ and 0.986 respectively (Figure 1). When we comparison between group A and group B using the mean $\mathrm{Ea}$ as a parameter of diastolic function of the pulsed wave tissue Doppler showed a cutoff value of $5.38 \mathrm{~cm} / \mathrm{sec}$ or more for prediction of recovery of diastolic function after myocardial infarction treated with 1ry PCI with a sensitivity and specificity of $97.4 \%$ and $100 \%$ respectively while the positive predictive value, negative predictive value and accuracy were $97.5 \%, 100 \%$ and 0.986 respectively (Figure 2 ).

\section{PTD measurements and global LV function}

In patients of group A (78\%), the mean LV EF increased from $46.8 \pm 6.3 \%$ at baseline to $59.3 \pm 6.2 \%$ after PCI $(\mathrm{P}<0.001)$, while in group $\mathrm{B}$, the mean LV EF of this group increased from $37.3 \pm 3.5$ at baseline to $39.2 \pm 3.4$ (mean $1.909 \%$ ) which is less than $5 \%$ (the cutoff value for prediction LV functional recovery). The mean value of $\mathrm{S}$-wave velocity in group A, showed significant improvement, that increased from $5.5 \pm 0.28$ at baseline study to $9.9 \pm 0.72 \mathrm{~cm} / \mathrm{s}$ one month 
after PCI ( $p<0.001)$, but it was insignificant in group B ( $p>0.05)$. The change in mean S-wave velocity before and after PCI was correlated with the change in global systolic function $(\mathrm{r}=0.003$, P-value $=0.034)$ (Figure 3) (Table 3).

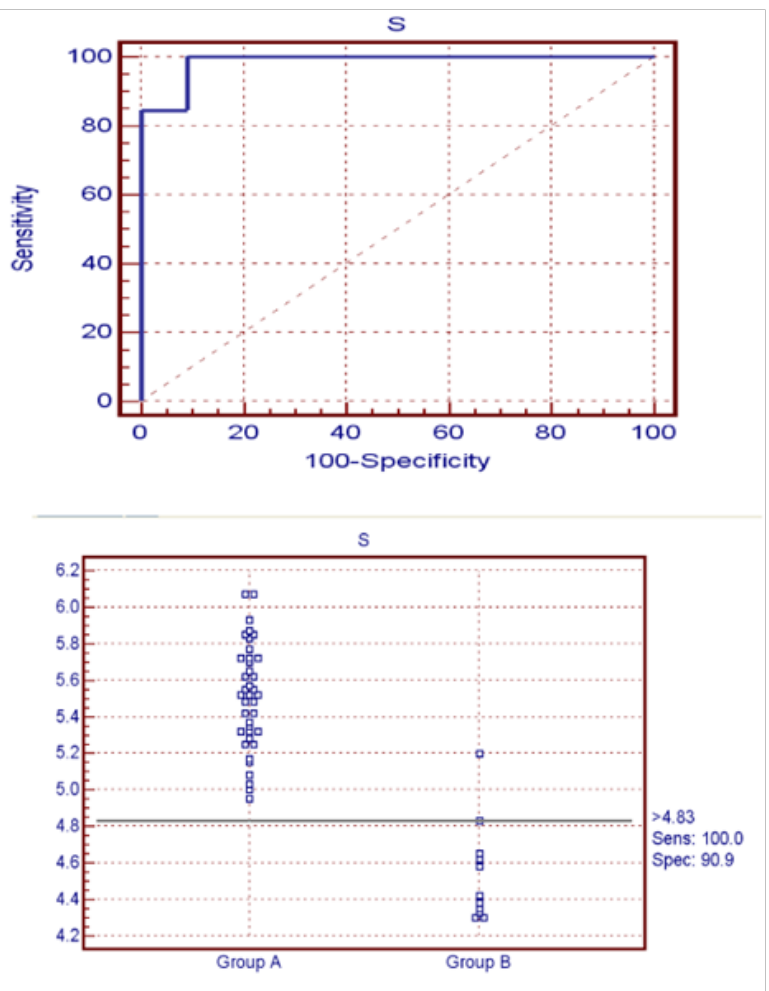

Figure I The sensitivity and specificity of S wave for detection of recovery of LV systolic function.

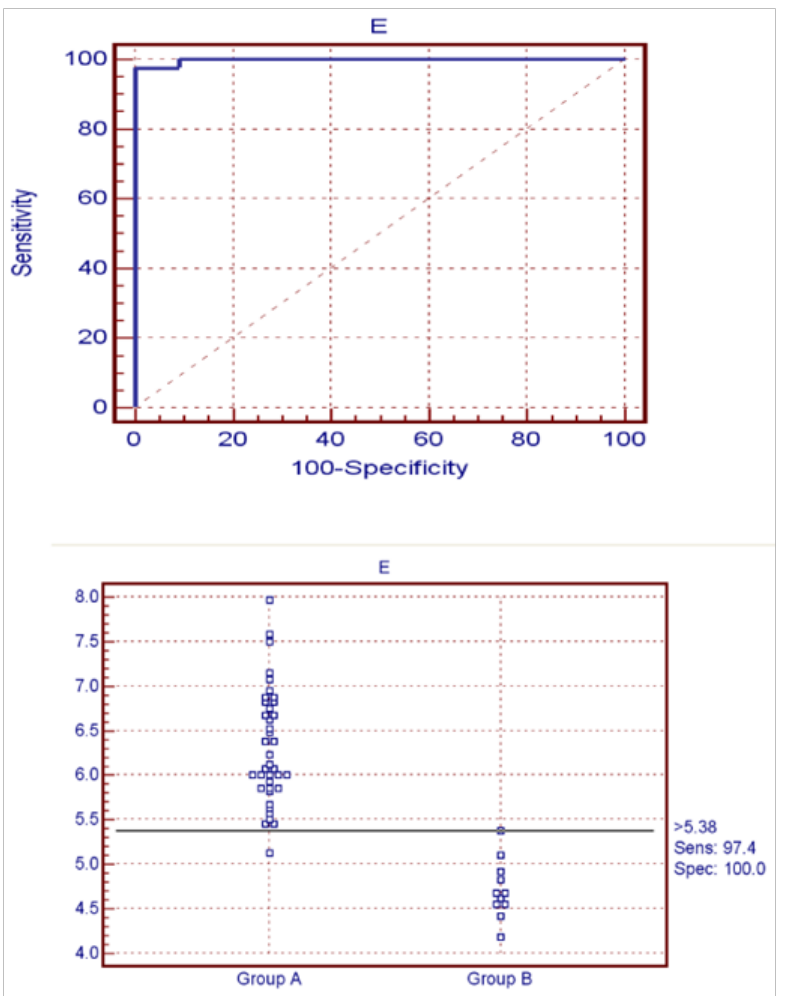

Figure 2The sensitivity and specificity of Ea for detection of diastolic function recovery.

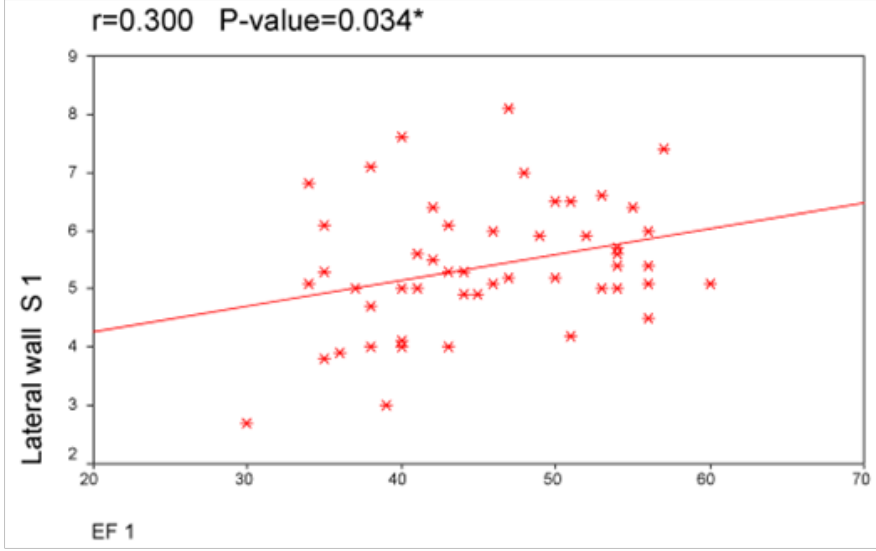

Figure 3 Relationship between mean S-wave velocity and LV EF.

Table 3 comparisons between the baseline and follow up Echocardiography data of the studied groups

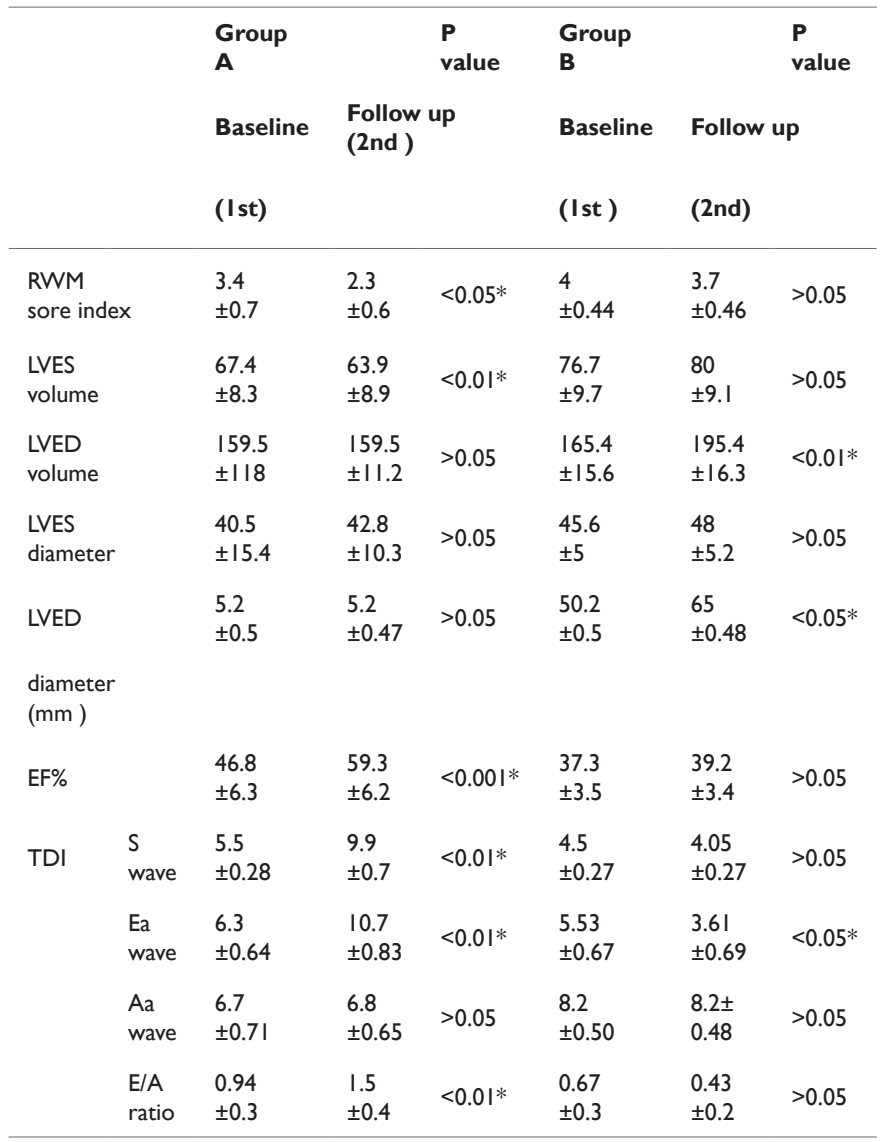

Follow up the patients for major adverse cardiac events

We found that, out of 11 patients in group B, 2 patients developed heart failure and required CCU admission, and one patient needed coronary revascularization with total MACE (27.27\%).

\section{Discussion}

The relationship between PTD parameters and noninvasive reperfusion criteria has been previously reported in patients who received thrombolytic therapy and thus, invasive markers of reperfusion were not evaluated. ${ }^{3}$ The success of reperfusion in the 
patients enrolled in our study was examined by a variety of invasive (TIMI flow grade and myocardial blush grade) and noninvasive (STsegment resolution and early peaking of cardiac biomarkers) markers. Of these, MBG and S-T segment resolution were specifically targeted, as these parameters also provide information about myocardial perfusion. ${ }^{10} \mathrm{We}$ recorded systolic and diastolic PW-TDI parameters at the level of the mitral annulus, evaluating the longitudinal shortening of the LV within 48 hours as baseline and one month later as a follow up study. As the LV contracts, shortening occurs along both the long and the short axis. Shortening across the LV long axis can be used in the evaluation of regional and global LV functions. ${ }^{11}$ In our study, patients with greater than $50 \%$ ST resolution showed significant improvement of systolic PTD parameters in the infarct-related and non-infarct related walls between the first and second PTD evaluation, whereas those with $50 \%$ or less S-T resolution showed significant deterioration in the systolic $\mathrm{S}$ wave values at all examined walls both at the baseline and follow up evaluation. Because of failure of micro-vascular reperfusion in these patients, they possibly demonstrate a larger infarct size, resulting in functional deterioration of systolic motion in the infarct-related segments. Similarly, patients with an early CK peak showed significant improvement of systolic PTD parameters and those with a delayed CK peak showed significant deterioration in the infarct-related walls.

In the present study, the mean, systolic (S) velocity showed a cutoff value of $4.83 \mathrm{~cm} / \mathrm{sec}$ or more for prediction of recovery of global systolic function with a sensitivity and specificity of $100 \%$ and $90 \%$ respectively, while the mean Ea showed a cutoff value of $5.38 \mathrm{~cm} / \mathrm{sec}$ or more for prediction of recovery of diastolic function after myocardial infarction treated with 1ry PCI with a sensitivity and specificity of $97.4 \%$ and $100 \%$ respectively. These simple noninvasive tools could play an important role in detecting the success of micro-vascular reperfusion through the changes detected in regional myocardial function after acute MI. In our study, all patients of studied group had diastolic dysfunction before PCI, that originated primarily from reduction of the Ea wave without change in Aa wave, because E-wave represents the energy dependant rapid filling phase and A-wave the atrial contraction dependent passive filling phase, it is not surprising that only E-wave peak levels are reduced. ${ }^{12}$ During acute ischemia, in order to protect LV filling against sudden reduction in LV compliance, A-wave peak levels can increase, which shows increased atrial contribution to ventricular filling. On the other hand, Alam et al. ${ }^{11}$ did not observe significant changes in A-wave peak levels 2 to 4 days after AMI compared with controls, which parallel to our finding.

Bach et al. ${ }^{13}$ Observed impairment of diastolic functions while systolic functions were preserved in the first 2 second after inflation of the balloon, although there was no significant $(50 \%)$ stenosis in remote myocardial segments, they concluded that, diastolic function is much more sensitive to ischemia than systolic function. In our study, EF was significantly higher in patients with good reperfusion criteria the mean LV EF of the group A increased from $46.8 \pm 6.38 \%$ before PCI to $59.3 \pm 6.2 \%$ after PCI $(\mathrm{P}<0.001)$. Although we have measured LVEF only 48 hours after the acute MI, such large-scale studied as TEAM or GUSTO have not found significant changes in LVEF in the period between 90 minutes and 1-to 2-week after AMI. ${ }^{14}$ In this study we found that, a significant positive correlation between LVEF and the average of systolic velocity measured from six different mitral annular locations ( $\mathrm{r}=0.300, \mathrm{p}$-value 0.034$)$. The average of systolic mitral annular velocity measured from six locations $4.83 \mathrm{~cm} / \mathrm{sec}$, can be used for prediction of recovery (increase of LVEF $\geq 5 \%$ between baseline and follow up) of global systolic function with a sensitivity and specificity of $100 \%$ and $90 \%$ respectively while the positive predictive value, negative predictive value and accuracy were $97.5 \%$, $100 \%$ and 0.986 respectively.

In a cross-sectional setting, positive correlations have been found between systolic myocardial velocities and LVEF. For example, Gulati et al. ${ }^{15}$ showed in a population with various underlying cardiac diseases that pulsed wave derived peak systolic velocity, measured as an average from six sites at the level of the mitral annulus correlated well $\left(\mathrm{r}^{1 / 4}\right.$ 0.85) with LVEF. However, no studies have investigated whether myocardial and/or annular velocities are related to LVEF at a time point when LVEF is generally accepted to have stabilized. ${ }^{16}$ In our study we found that, the predictors of LV function recovery after PCI were, LVEF $>45 \%$, LVES volume $<68 \mathrm{ml}$, and $\mathrm{S}$ wave $>5.5 \mathrm{~cm} /$ sec. in baseline echocardiography. In a study of Joost et al. ${ }^{17}$ in which they aimed to detect if TDI can predict LV function recovery, infarct size and remodeling as measured by MRI at 4 months, they used the screening ability of $\mathrm{S}$ wave to identify patients with LVEF greater or less than $40 \%$ (area under the curve $0.79 ; \mathrm{P}^{1} / 40.009$ ). Although numbers were low for patients with poor LVEF, S velocity $\leq 3.0 \mathrm{~cm} / \mathrm{s}$ appeared to be an excellent predictor to identify post-MI patients with $\mathrm{LVEF} \leq 40 \%$ (PPV $100 \%$ ), while $\mathrm{S}$ wave $6.5 \mathrm{~cm} / \mathrm{s}$ or more excellent to identify post-MI patients with $\mathrm{LVEF} \geq 40 \%$ with sensitivity $62 \%$, whereas the specificity was $78 \%$. Park et al. ${ }^{18}$ found that, $46 \%$ of patients showed remodeling, in contrast to $18 \%$ of the patients in Joost et al. ${ }^{17}$ study, while in our study LV remodeling was detected in $22 \%$ of patients who had failed to show significant invasive and non-invasive reperfusion. This difference can be explained by the difference in percentage of anterior infarctions included ( $100 \%$ vs. $52 \%$, vs $50 \%$ respectively). Infarct size on contrast MRI may be superior to LVEF and LV volumes for predicting long-term mortality in patients with MI Roes et al. ${ }^{19}$ they established a firm inverse relationship between $\mathrm{S}$ and the extent of myocardial necrosis at 4 months, even with same age and sex. We found that, out of 11 patients, three patients $(27.27 \%)$ in group B were developed MACE, 2 of them admitted to CCU by heart failure while the other one need for revascularization due to recurrent chest pain.

\section{Conclusion}

LV recovery occurs in STEMI patients treated with primary $\mathrm{PCI}$ and it is an important for favorable outcome and can be estimated by TDI.

\section{Acknowledgement}

None

\section{Conflicts of interest}

Authors declare that there is no conflict of interest.

\section{References}

1. Zijlstra F, Hoorntje JC, de Boer MJ, et al. Long-term benefit of primary angioplasty as compared with thrombolytic therapy for acute myocardial infarction. N Engl J Med. 1999;341(19):1413-1419.

2. Bolognese L, Neskovic AN, Parodi G, et al. Left ventricular remodeling after primary coronary angioplasty: patterns of left ventricular dilation and long-term prognostic implications. Circulation. 2002;106(18):23512357.

3. Iyisoy A, Amasyali B, Kilic A, et al. Relationship between noninvasive 
reperfusion criteria and pulsed-wave tissue Doppler parameters in patients with acute myocardial infarction receiving thrombolytic therapy. Echocardiography. 2003;20(3):237-48.

4. Brodie BR, Stuckey TD, Hansen C, et al. Relation between electrocardiographic ST-segment resolution and early and late outcomes after primary percutaneous coronary intervention for acute myocardial infarction. Am J Cardiol. 2005;95(3):343-348.

5. Matetezky S, Friemark D, Choraqui P, et al. The distinction between coronary and myocardial reperfusion after thrombolytic therapy by clinical markers of reperfusion. J Am Coll Cardiol. 1998;32(5):13261330 .

6. Gibson CM, Cannon CP, Murphy SA, et al. Relationship of the TIMI myocardial perfusion grades, flow grades, frame count, and percutaneous coronary intervention to long term outcomes after thrombolytic administration in acute myocardial infarction. Circulation 2002;105(16):1909-1913.

7. Henriques JP, Zijlstra F, van’t Hof AW, et al. Angiographic assessment of reperfusion in acute myocardial infarction by myocardial blush grade. Circulation. 2003;107(16):2115-2119.

8. Edvardsen T, Urheim S, Skulstad H, et al. Quantification of left ventricular systolic function by tissue Doppler echocardiography: added value of measuring pre and post ejection velocities in ischemic myocardium. Circulation. 2002;105:2071-2077.

9. Raymond, Bayarri. P Values are not Error Probabilities. A working paper that explains the difference between Fisher's evidential p-value and the Ney man-Pearson Type I error rate $\alpha .2003$.

10. Poli A, Fetiveau R, Vandoni P, et al. Integrated analysis of myocardia blush and ST-segment elevation recovery after successful primary angioplasty. Circulation. 2002;106(3):313-318.

11. Alam M, Wardell J, Anderson E, et al. Effects of first myocardial infarction on left ventricular systolic and diastolic function with the use of mitral annular velocity determined by pulsed wave Doppler tissue imaging. $J A m$ Soc Echocardiogr. 2000;13(5):343-352.
12. Gorcsan J III, Gulati VK, Mandarino WA, et al. Color coded measures of myocardial velocity throughout the cardiac cycle by tissue Doppler imaging to quantify regional left ventricular function. Am Heart $J$ 1999;131(6):1203-1213.

13. Bach DS, Armstrong WF, Donovan CL, et al. Quantitative Doppler tissue imaging for assessment of regional myocardial velocities during transient ischemia and reperfusion. Am Heart J. 1966;132(4):721-725.

14. Anderson JL, Karagounis LA, Califf RM. Met analysis of five reported studies on the relation of early coronary patency grades with mortality and outcomes after acute myocardial infarction . Am J cardiol. 1966;78(1):18.

15. Gulati VK, Katz WE, Follansbee WP, et al. Mitral annular descent velocity by tissue Doppler echocardiography as an index of global left ventricular function. Am J Cardiology. 1966;77(11):979-984.

16. Dill T, Schachinger V, Rolf A, et al. Intracoronary administration of bone marrow derived progenitor cells improves left ventricular function in patients at risk for adverse remodeling after acute ST-segment elevation myocardial infarction: results of the Reinfusion of Enriched Progenitor cells And Infarct Remodeling in Acute Myocardial Infarction study (REPAIR-AMI) cardiac magnetic resonance imaging sub-study. Am Heart J. 2009;157(3):541-547.

17. Van Melle JP, Van der Vleuten PA, Hummel YM, et al. Predictive value of tissue Doppler imaging for left ventricular ejection fraction, remodeling, and infarct size after percutaneous coronary intervention for acute myocardial infarction. Eur J Echocardiogr. 2010;11(7):596-601.

18. Park YH, Kang SJ, Song JK, et al. Prognostic value of longitudinal strain after primary reperfusion therapy in patients with anterior-wall acute myocardial infarction. J Am Soc Echocard. 2008;21(3):262-267.

19. Roes SD, Kelle S, Kaandorp TA, Kokocinski T, et al. Comparison of myocardial infarct size assessed with contrast-enhanced magnetic resonance imaging and left ventricular function and volumes to predict mortality in patients with healed myocardial infarction. Am J Cardiol. 2007;100(6):930-936 